\title{
Monte Verde. Un Asentamiento Humano del Pleistoceno Tardio en el Sur de Chile, Tom Dillehay, Colección Serie Universitaria, LOM Ediciones, Universidad Austral de Chile, 2004, pp. 173, Santiago.
}

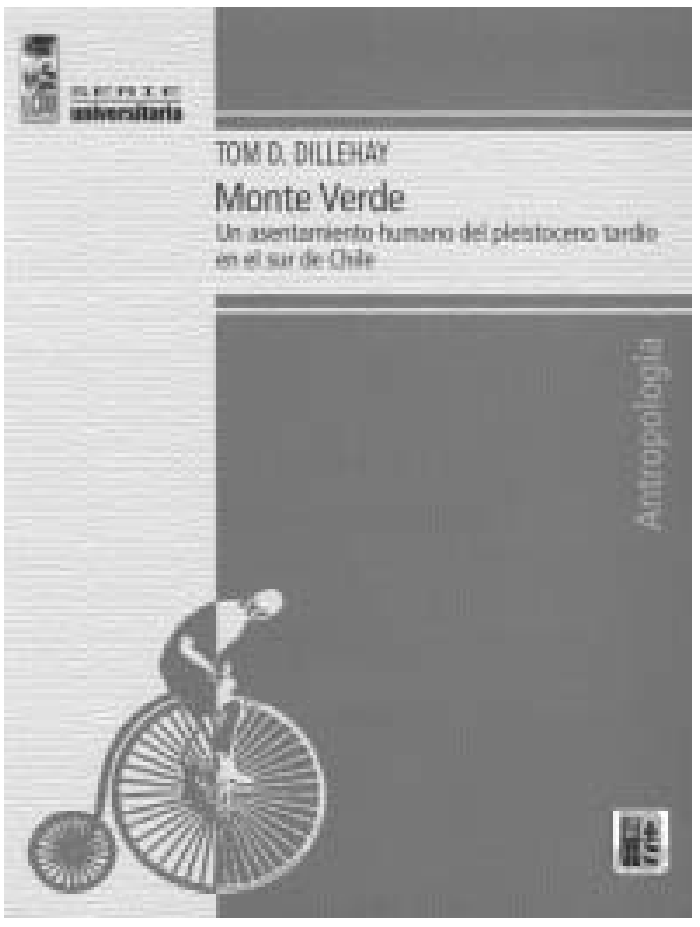

\section{Comentario de Gustavo G. Politis *}

Este libro es una síntesis de las investigaciones llevadas a cabo por Tom Dillehay y un numeroso grupo interdisciplinario de científicos en el sitio Monte Verde, en el sur de Chile. Es la versión reducida de los dos volúmenes publicados en inglés por la Smithsonian Institution Press en 1989 y 1997. Ambos volúmenes causaron un alto impacto en el debate sobre el poblamiento americano y sentaron las bases para una renovada discusión que giró sobre dos ejes: una antigüedad mayor a 12.000 años a.p. y una forma de vida distinta de la inferida para los cazadores Clovis. Si bien para el primer tema ya habían suficientes datos de otros sitios como para proponer una antigüedad pre-Clovis en América del Sur (como lo refleja una discusión ya clásica, ver por ejemplo Bryan 1973, 1975; Bryan et al. 1978; Cardich et al. 1973; Haynes 1974; Lynch 1974; Ochsenius y Gruhn 1979); el segundo aspecto tuvo un aporte signifi- cativo con los datos obtenidos en Monte Verde. En este sitio se evidencia que hace unos 13.000 años a.p. algunos de los grupos humanos que habitaban América del Sur tenían una economía generalizada basada tanto en la recolección de plantas como en la caza de grandes animales. Y que además ocupaban sus campamentos durante períodos mucho más largos que los inferidos para otros sitios contemporáneos.

Los dos volúmenes en inglés estuvieron acompañados de un duro debate en diferentes ámbitos (congresos, revistas científicas de primer nivel, ver por ejemplo entre muchos otros, Adovasio y Pedler 1997; Anderson 1999; Fiedel 1999; Meltzer 1999; West 1993) y hasta se organizó una visita de expertos al sitio que pretendió resolver la polémica in situ y llegar a una especie de acuerdo final (ver Gore 1997; Meltzer et al. 1997). Como ya he expuesto en otro artículo (Politis 1999) la sola opinión de un puñado de expertos no parece ser la manera más adecuada de validar hallazgos ni de confirmar hipótesis. El consenso sobre la aceptación de las hipótesis y modelos propuestos por Dillehay y colaboradores debe llegar de la lectura detallada, objetiva y desapasionada de las evidencias, las que, afortunadamente están pormenorizadas en los dos volúmenes en inglés y resumidas en el texto en castellano.

Sin embargo, una de las principales discusiones se produjo cuando Fiedel (1999), luego de una lectura crítica del segundo libro (el de 1997), notó una serie de inconsistencias que lo llevaron a plantear serias dudas sobre el sitio y a reactivar nuevamente el debate. Debido a esto, Dillehay, Collins y el resto de los investigadores del proyecto se dieron cuenta que las correcciones que los diferentes autores de los capítulos habían realizado a las pruebas de imprenta, en el examen final del libro, nunca habían sido incorporadas a la versión impresa. Afortunadamente, Smithsonian Institution Press publicó un tercer texto de "Erratas" en donde se subsanaron más de 950 errores grandes y se clarificaron las principales dudas de Fiedel.

En el libro en español, objeto de este comentario, se explica todo este proceso y, aunque el detalle de la información presentada es mucho menor, se aclaran los principales errores que tenía el segundo volumen. Ese es uno de los aportes originales de la obra ya que presenta un producto compacto, resumido y corregido de

CONICET- Universidad Nacional del Centro de la Pcia. de Buenos Aires y Universidad Nacional de La Plata, Argentina. gpolitis@fcnym.unlp.edu.ar 
los dos libros originales en inglés. La otra contribución de esta síntesis en castellano es que pone a disposición de un público más amplio la información relevante del sitio y las hipótesis centrales generadas por el equipo multidisciplinario de investigación. Esto entonces tiene un doble valor: llegar más allá de los especialistas (lo que es cada vez más necesario en la arqueología contemporánea) y a la vez poner en español, a disposición de estudiantes e investigadores, los resultados obtenidos en uno de los sitios más importantes para discutir el poblamiento americano.

Este libro resume los aspectos más relevantes de Monte Verde: los patrones arquitectónicos, los rasgos culturales, los análisis líticos y faunísticos, el paleoambiente y la cronología. De esta manera, se puede acceder a descripciones relativamente completas y amenas sin tener que internarse en los capítulos originales de los volúmenes en inglés que, a veces, son demasiado detallados y asequibles sólo para superespecialistas.

Otro punto importante del libro es que está muy bien ilustrado. Las fotos son abundantes, a color y se notan muy bien los detalles. Esto es particularmente significativo en un sitio como Monte Verde, con un registro atípico, porque permite evaluar las características de los rasgos arquitectónicos, del material lítico y de los instrumentos de madera. Así, las ilustraciones son de gran ayuda para que el lector pueda evaluar por sí mismo las interpretaciones de Dillehay y colaboradores. Hay también mapas, perfiles y croquis que completan la información presentada. Por último, debe remarcarse que la traducción y edición en español de esta síntesis estuvo a cargo de la arqueóloga uruguaya Cecilia Mañosa, quien figura como colaboradora. El trabajo de Mañosa es muy bueno y el texto no tiene errores. La lectura es ágil y se han traducido adecuadamente los términos técnicos que frecuentemente se prestan a confusión.

Para terminar con esta reseña deseo expresar que quienes quieran conocer en detalles aspectos específicos de la investigación de Monte Verde deben ir a los dos volúmenes en inglés. Este libro en español no los reemplaza. Sin embargo, es una muy buena síntesis de uno de los sitios más importantes para entender el poblamiento americano. Y el hecho de que esté en español $\mathrm{y}$ en un lenguaje ameno y accesible permite su discusión en clases y lo proyecta hacia un público más amplio. De alguna manera, contribuye a disminuir esa brecha que existe entre América del Norte y América del Sur en la generación, difusión y validación de la información arqueológica.

\section{Referencias Citadas}

Adovasio, J. y D. R. Pedler

1997 Monte Verde and the antiquity of humankind in the Americas. Antiquity 71:573-80.

Anderson, D.

1999 Monte Verde and the way american archaeology does business. Discovering Archaeology 1 (6). Special report 19-20. Bryan, A.

1973 Paleoenvironmental and cultural diversity in Late Pleistocene South America. Quaternary Research 3:237-256.

1975 Paleoenvironmental and cultural diversity in Late Pleistocene South America. A rejoinder to Vance Haynes and a reply to Thomas Lynch. Quaternary Research 5:151-159.

Bryan, A., R. Casamiquela, J. M. Cruxent, R. Gruhn y C. Ochsenius 1978 An El Jobo mastodon kill at Taima-taima, Venezuela. Science 200:1275-77.

Cardich, A., L. A. Cardich y A. Hajduk

1973 Secuencia arqueológica y cronología rediocarbónica de la Cueva 3 de Los Toldos (Santa Cruz, Argentina). Relaciones de la Sociedad Argentina de Antropología 7 (n.s.):85-123.

Dillehay, $\mathrm{T}$.

1989 Monte Verde. Late Pleistocene Settlement in Chile. Volume 1. Paleonvironment and Site Context. Smithsonian Institution Press, Washington and London.

1997 Monte Verde. A Late Pleistocene Settlement in Chile. Volume 2. The Archaeological Context and Interpretation. Smithsonian Institution Press, Washington and London.

Fiedel, S.

1999 Artifact provenience at Monte Verde: Confusion and contradictions. Discovering Archaeology 1(6). Special Report: Monte Verde Revisited: 1-12.
Gore, R.

1997 The most ancient americans. National Geographic 192:92-99.

Haynes, C. V.

1974 Paleoenvironment and cultural diversity in the Late Pleistocene South America: A reply to A. L. Bryan. Quaternary Research 4:378-382.

Lynch, T.

1974 Early man in South America. Quaternary Research 4:356-377.

1990 Glacial-age man in South America: A critical review. American Antiquity 55:12-36.

Meltzer, D.

1999 On Monte Verde. Discovering Archaeology 1(6). Special Report: Monte Verde Revisited: 16-17.

Meltzer, D., D. Grayson, G. Ardila, A. Barker, D. Dinkauze, C. V. Haynes, F. Mena, L. Núñez y D. Stanford

1997 On the Pleistocene antiquity of Monte Verde, Southern Chile. American Antiquity 62:659-63.

Ochsenius, C. y R. Gruhn, editores

1979 Taima-taima. A Late Pleistocene Paleo-Indian kill site in Northwestern South America -Final Reports of the 1976 Excavations-. South American Quaternary Docummentation Program. República Federal Alemana.

West, F.

1993 Review of palaeoenvironment and site content at Monte Verde. American Antiquity 58:166-167. 
